Arq. Bras. Med. Vet. Zootec., v.70, n.5, p.1655-1659, 2018

\title{
Comunicação
}

[Communication]

\section{Um caso raro de destruição total do pâncreas por pancreatite em cão}

[A rare case of total pancreatic destruction due to pancreatitis in a dog]

\author{
B.O.S. Câmara, F.A.B. Viana, B.N.T. Ribeiro, N.M. Ocarino, \\ A.C. Nepomuceno, R. Serakides*
}

Escola de Veterinária - Universidade Federal de Minas Gerais - Belo Horizonte, MG

A pancreatite é uma doença frequente em cães, que se caracteriza por sinais gastrointestinais discretos e intermitentes, podendo se apresentar nas formas aguda ou crônica. Ela caracteriza-se por infiltrado inflamatório e fibrose, com consequente perda gradual do parênquima pancreático e progressiva perda das funções endócrina e exócrina (Watson, 2012). Já foram relatados outros casos de destruição do pâncreas em cães. No entanto, nesses casos foi possível encontrar, por exame microscópico, resquícios do parênquima pancreático e de células inflamatórias (Watson et al., 2010a). $\mathrm{Na}$ medicina, há um relato de necrose total do pâncreas decorrente de hipotermia terapêutica provocada, mas sem relação com processo inflamatório (Ura et al., 2002). Assim, o objetivo deste trabalho é relatar um caso raro de destruição total do pâncreas causada por pancreatite em uma cadela adulta.

Uma cadela da raça Poodle, com 16 anos de idade, foi encaminhada ao Hospital Veterinário da Universidade Federal de Minas Gerais (UFMG) por apresentar prostração intensa durante uma semana, anorexia e vômito súbito. Ela já havia sido diagnosticada com epilepsia idiopática há 13 anos, além de apresentar catarata e doença periodontal grave. Ao exame clínico, apresentou dor abdominal discreta, desidratação e hiperglicemia de $448 \mathrm{mg} / \mathrm{dL}$, sendo os valores de referência de glicemia para essa espécie de 75 a 120mg/dL (Kumar et al., 2014). O animal foi internado com suspeita de cetoacidose diabética.

Recebido em 13 de junho de 2017

Aceito em 9 de fevereiro de 2018

*Autor para correspondência (corresponding author)

E-mail: serakidesufmg@ gmail.com
Durante o período de internação, a cadela apresentou também poliúria e polidipsia.

Realizaram-se os exames de hemograma, perfil bioquímico, urinálise e hemogasometria. A hemogasometria demonstrou a presença de uma acidose metabólica ( $\mathrm{pH} 7,1$ e $\mathrm{HCO} 3$ 12mmol/L) e hipercalemia discreta $(5,6 \mathrm{mEq} / \mathrm{L})$. A urinálise revelou glicosúria $(++)$, cetonúria $(+)$ e proteinúria, $\mathrm{pH} 8$ e raros cocos. $\mathrm{O}$ hemograma revelou leucocitose (22.300) por neutrofilia, com neutrófilos tóxicos (corpúsculos de Dohle), neutrófilos hipersegmentados e trombocitose (709.000). O segundo hemograma, realizado 48 horas após hidratação na internação, revelou a presença de anemia (hematócrito 29\%). O perfil bioquímico mostrou aumento de enzimas hepáticas (ALT de 229,3; AST de 149,98; FA de 261,19), hiperglicemia e hiperalbuminemia $(4,58)$.

Foi realizada também ultrassonografia abdominal, na qual se observou tecido adjacente à topografia pancreática espessado e hiperecogênico (reativo), associado a plissamento de duodeno e discreta quantidade de líquido local, indicativo de peritonite focal, além de pâncreas hipoecogênico e reduzido de tamanho $(0,6 \mathrm{~cm}$ de espessura), sinais indicativos de pancreatite (Fig. 1). O recurso Doppler utilizado revelou ausência de vascularização na região pancreática.

Após sete dias de internação, com reposição hidroeletrolítica e controle da glicemia, a cadela recebeu alta, tendo como prescrição a insulina 
intermediária $\quad(\mathrm{NPH}) . \quad$ No entanto, aproximadamente 30 dias após, ela foi novamente internada, desta vez por um quadro de status epilepticus. O proprietário revelou dificuldade no controle glicêmico em casa e detecção de episódios de hipoglicemia por mensuração com glicosímetro. Ao exame clínico, a cadela apresentou prostração intensa e ausência de dor à palpação abdominal, com glicemia menor que a mínima detectável, tendo sido encaminhada ao setor de emergência do Hospital Veterinário da UFMG. Devido ao insucesso no controle das crises convulsivas, com perda de alguns reflexos e formação de edema pulmonar, foi realizada a eutanásia do animal.

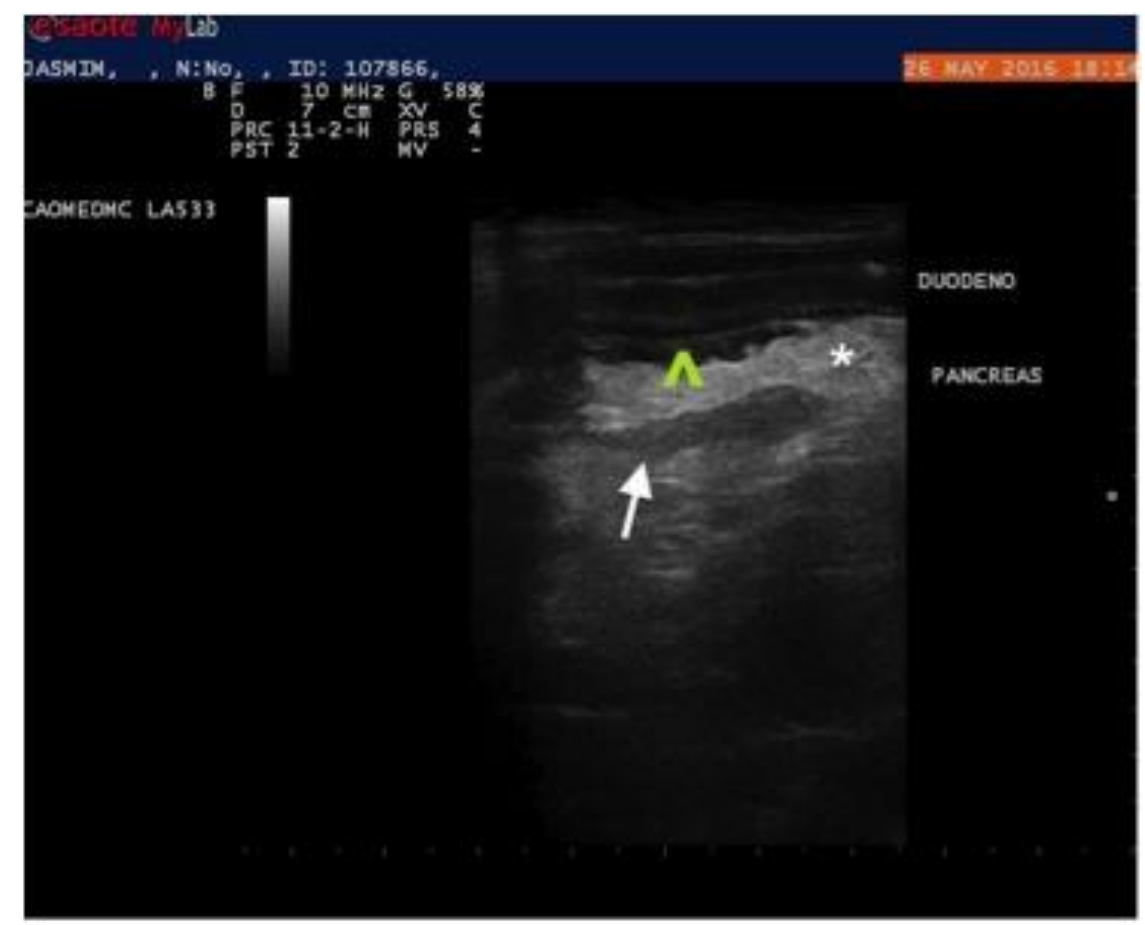

Figura 1. Imagem ultrassonográfica de abdômen de cadela Poodle, 16 anos. Nota-se pâncreas hipoecogênico e reduzido de tamanho (seta), plissamento de duodeno (cabeça de seta) e reatividade tecidual adjacente (asterisco). Imagem compatível com pancreatite.

O animal foi encaminhado à necropsia, na qual não foi encontrado qualquer resquício do pâncreas. Além disso, as mucosas ocular, oral e a musculatura estavam intensamente pálidas e os pulmões apresentavam hiperemia e edema moderados. O fígado estava discretamente aumentado e amarelado, com evidenciação do padrão lobular e discretamente friável. Os rins estavam discretamente diminuídos de tamanho, hiperêmicos e com superfície moderadamente irregular.

Todo o tecido adiposo da região próxima ao duodeno, correspondente ao local do pâncreas, foi coletado e foram realizados cortes histológicos seriados. Microscopicamente, apenas uma estrutura de origem pancreática, semelhante a um ducto, foi localizada no tecido adiposo, como focos de infiltrado inflamatório misto discreto, constituído por neutrófilos, macrófagos e linfócitos. Em algumas áreas, foram observados restos celulares e fibrina próximos à reação inflamatória (Fig. 2). Os rins apresentavam nefrose multifocal a coalescente moderada e glomerulonefrite membranosa moderada; no fígado, havia degeneração multifocal discreta. Nos demais órgãos, não se observou nenhuma alteração digna de nota.

As alterações do perfil bioquímico e da urinálise são compatíveis com os achados macro e microscópicos evidenciados. Nos seres humanos, a anemia é uma complicação da diabetes comum e frequentemente não é identificada. Nesta espécie, a anemia é mais frequente e mais severa 
nos indivíduos com diabetes que naqueles sem diabetes mellitus, independentemente da taxa de filtração glomerular deles. A etiologia da anemia nesses pacientes pode incluir uma deficiência ou ineficácia na atuação da eritropoetina, deficiência nutricionais, inflamação sistêmica, medicações ou desordens imunomediadas. Dessa forma, a nefrose e a glomerulonefrite encontradas na necropsia da cadela em estudo, bem como a presença de uma anemia no segundo hemograma, ainda que este animal não tivesse histórico de alteração e perda de função renal prévias, poderiam estar relacionadas (Sarafian et al., 2010). Além disso, a presença de uma degeneração multifocal em fígado e o aumento de enzimas hepáticas no perfil bioquímico coletado no momento da internação apontam para a existência de uma lesão hepática na cadela. Segundo Watson et al. (2010b), o aumento de enzimas hepáticas é um achado comum na pancreatite crônica, de modo que uma hepatite reativa coexiste com a última, possivelmente em consequência da drenagem de toxinas e de mediadores inflamatórios no sangue portal provenientes de um pâncreas doente. A presença de uma leucocitose por neutrofilia nos hemogramas da cadela, juntamente com as imagens de ultrassom obtidas, corroboram a ideia de que o animal apresentava um processo inflamatório crônico, porém ativo e agudizado, no pâncreas (Mansfield, 2012; Bostrom et al., 2013). A presença de uma hiperglicemia significativa, associada a glicosúria, cetonúria e acidose metabólica, aponta para a teoria de que o animal em estudo possuía a doença diabetes mellitus descompensada, encontrando-se num estado de cetoacidose diabética (Hume et al., 2006).
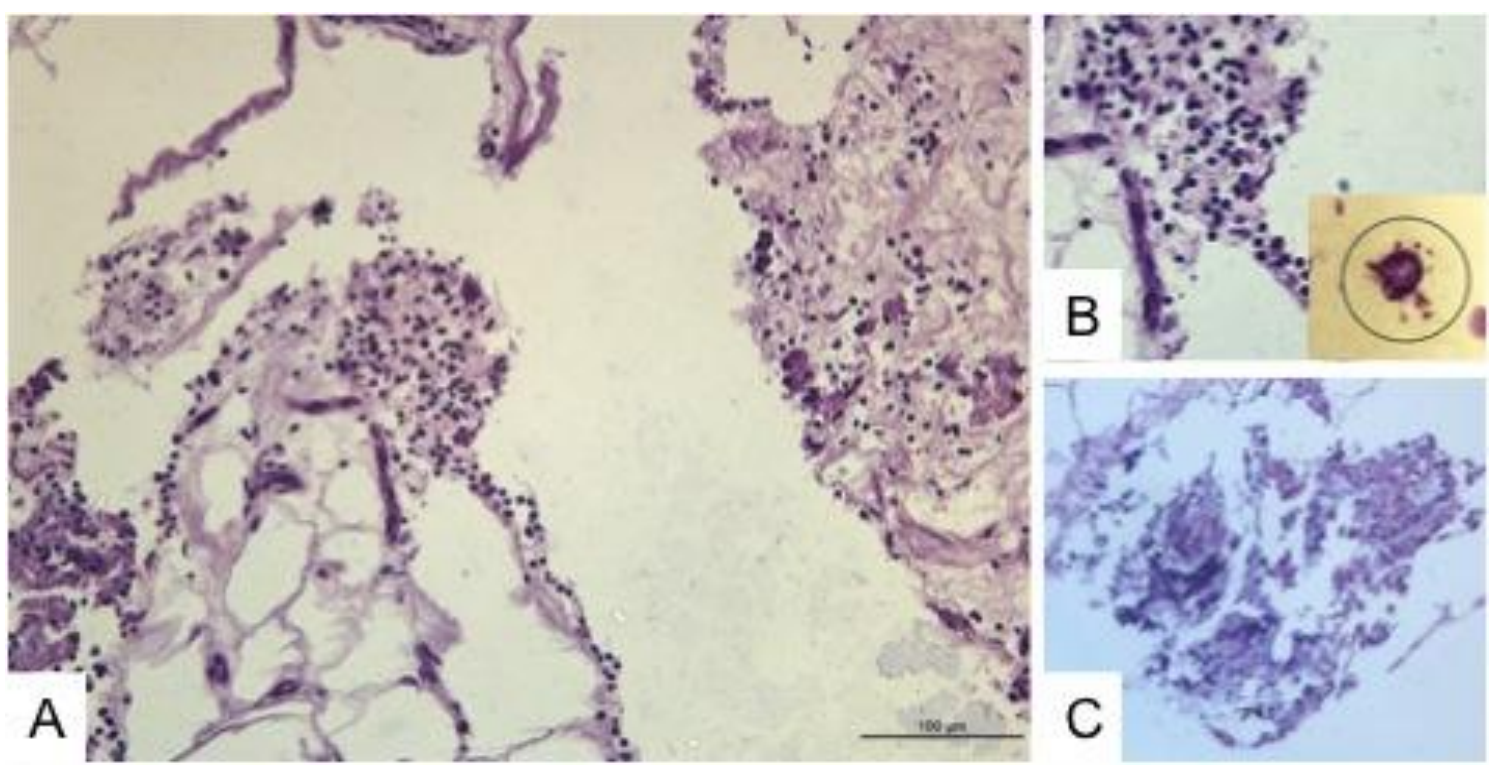

Figura 2. As Fig. A, B e C apresentam diferentes regiões de tecido adiposo da topografia pancreática, no qual há infiltrados inflamatórios. O quadro menor mostra estrutura pancreática remanescente, identificada como ducto pancreático (círculo).

No sistema nervoso central, não havia alterações macro ou microscópicas. Soma-se a isso o fato de que convulsões não sāo geralmente identificadas como sintomas de pancreatite (Bostrom, 2012; Mansfield 2012; Watson, 2012) e de diabetes mellitus (Catchpole et al., 2005; Kumar et al., 2014), bem como o de que a epilepsia previamente diagnosticada estava controlada há anos. Dessa forma, o quadro de crises epilépticas apresentado pela cadela poderia estar relacionado à dificuldade no controle glicêmico do animal, tanto no ambiente de internação quanto em sua residência. Essa dificuldade se deve, provavelmente, à presença de fatores causadores de resistência à insulina, dentre eles a pancreatite, estando a ação da insulina reduzida neste caso (Hess, 2010). Assim, uma hipoglicemia intensa, como a apresentada pelo animal, pode causar fraqueza, letargia, ataxia, confusão e crises convulsivas (Bennett, 2002). 
A ultrassonografia abdominal é o exame de imagem normalmente utilizado para identificação da pancreatite. A pancreatite aguda necrosante pode ser frequentemente identificada pela presença de uma hiperecogenicidade no mesentério da topografia pancreática, de um pâncreas hipoecoico e aumentado, de plissamento de duodeno e de acúmulo de líquido próximo ao órgão, indicando uma peritonite focal (Mansfield, 2012). Já a pancreatite crônica caracteriza-se por redução no tamanho do pâncreas e com presença ou não de hiperecogenicidade dele (Waton et al., 2011). No animal aqui estudado, uma área hipoecoica de $0,6 \mathrm{~cm}$ de espessura foi identificada pela equipe de diagnóstico por imagem do Hospital Veterinário da UFMG como sendo o pâncreas reduzido de tamanho. Além disso, fatores como reatividade tecidual adjacente, líquido livre no local, plissamento de duodeno e recurso Doppler com ausência de vascularização na região pancreática apontaram para a existência de um processo inflamatório do órgão. Tais características sustentam a hipótese de que a cadela tenha apresentando um quadro de agudização de um processo de pancreatite crônica. Não é incomum em cães que a pancreatite crônica se apresente inicialmente como um quadro agudo, após um longo período de doença subclínica com destruição de grande parte do parênquima pancreático (Watson, 2012). O médico veterinário pode não suspeitar da existência de uma pancreatite crônica até o animal atingir o estágio final da doença ou desenvolver diabetes mellitus, como se acredita ser o caso desta cadela.

Há forte associação entre a pancreatite e a diabetes mellitus. No entanto, existe uma complicada relação de causa e efeito, já que, em alguns casos, uma diabetes mellitus de outra causa predispõe à ocorrência da pancreatite por uma hiperlipidemia por ela gerada, enquanto em outros, a diabetes mellitus parece ser consequência da perda de ilhotas pancreáticas num estágio final da pancreatite crônica. Tornase difícil, portanto, a definição dessa relaçāo de causa e efeito sem a histologia de fragmento de pâncreas (Xenoulis e Steiner, 2010; Watson, 2012). Desse modo, a diabetes mellitus não controlada, apresentada pela cadela em questão, poderia ter contribuído para a agudização da pancreatite crônica, resultando no quadro de cetoacidose diabética apresentado pelo animal.

$\mathrm{Na}$ cadela em estudo, verificou-se a presença de sinais característicos de pancreatite, bem como a visualização do pâncreas pelo exame ultrassonográfico. Dessa forma, a hipótese de uma aplasia de pâncreas foi totalmente afastada. Tal conclusão é também suportada pelo início tardio dos sintomas relacionados à inflamação e à diabetes mellitus.

Pelo conhecimento dos autores, esta comunicação aborda o primeiro caso de destruição total de pâncreas por pancreatite na espécie canina. Apenas um único caso de necrose total do pâncreas foi relatado, na medicina, consequente de uma hipotermia induzida. No entanto, o mecanismo de ação, neste caso, não parece ter sido inflamatório, tendo em vista a ausência de sinais característicos de inflamação do adjacente ao pâncreas à ultrassonografia (Ura et al., 2002).

Palavras-chave: cão, pancreatite, destruição do pâncreas

\begin{abstract}
A 16-year-old female Poodle entered UFMG's Veterinary Hospital with severe prostration, lack of appetite, and vomit. During physical examination, abdominal pain, dehydration, and hyperglycemia (448mg/dl) were found, therefore the animal was admitted under the suspicion of diabetic ketoacidosis. Screening revealed metabolic acidosis, hyperkalemia, glycosuria, ketonuria, and proteinuria. Leukocytosis, thrombocytosis, increase in the number of hepatic enzymes and hyperglycemia were also present in these tests. The ultrasound images showed a smaller and hypoechogenic pancreas, irregularity and folds in duodenum and reactivity of the surrounding tissue, indicating pancreatitis. Thirty days after the dog had been discharged for treatment at home, it was taken back to the veterinary hospital due to status epilepticus, which motivated the owner's decision of euthanasia. During post mortem examination no trace of pancreas was found. On histological examination of the adipose tissue next to the duodenum, only one pancreatic duct was seen, together with inflamatory cells, thus characterizing a rare case of total pancreatic destruction due to pancreatitis.
\end{abstract}

Keywords: dog, pancreatitis, pancreatic destruction 


\section{REFERÊNCIAS}

BENNETT, N. Monitoring techniques for diabetes mellitus in the dog and the cat. Clin. Tech. Small Anim. Pract., v.17, p.65-69, 2002.

BOSTROM, BM.; XENOULIS, P. G.; NEWMAN, S.J. et al. Chronic pancreatitis in dogs: a retrospective study of clinical, clinicopathological, and histopathological findings in 61 cases. Vet. J., v.195, p.73-79, 2013.

CATCHPOLE, B.; RISTIC, J.M.; FLEEMAN, L.M. et al. Canine diabetes mellitus: Can old dogs teach us new tricks? Diabetologia, v.48, p.1948-1956, 2005.

HESS, R.S. Insulin resistance in dogs. Vet. Clin. Small Anim., v.40, p.309-316, 2010.

KUMAR, P.; KUMARI, R.R.; KUMAR, M. et al. Current practices and research updates on diabetes mellitus in canine. Vet. World, v.7, p.952-959, 2014.

MANSFIELD, C. Acute pancreatitis in dogs: advances in understanding, diagnostics and treatment. Top. Comp. Anim. Med., v.27, p.123$132,2012$.

NEWMAN, S.J.; STEINER, J.M.; WOOSLEY, $\mathrm{K}$. et al. Histologic assessment and grading of the exocrine pancreas in the dog. J. Vet. Diagn. Investig., v.18, p.115-118, 2006.
SARAFIAN, D.A.; SHTEINSHNAIDER, M.; TZUR, I. et al. Anemia in diabetic patients at an internal medicine ward: clinical correlates and prognostic significance. Eur. J. Intern. Med., v.21, p.91-96, 2010.

URA, H.; ASAI, Y.; MORI, K. et al. Total necrosis of the pancreas and renal cortex secondary to hypothermia therapy. J. Trauma, v.52, p.987-989, 2002.

WATSON, P. Chronic pancreatitis in dogs. Top. Comp. Anim. Med., v.27, p.133-139, 2012.

WATSON, P.J.; ARCHER, J.; ROULOIS, A.J. et al. Observational study of 14 cases of chronic pancreatitis in dogs. Vet. Rec., v.167, p.968-976, 2010a.

WATSON, P.J.; ROULOIS, A.; SCASE, T. et al. Characterization of chronic pancreatitis in English cocker spaniels. J. Vet. Int. Med., v.25, p.797-804, 2011.

WATSON, P.J.; ROULOIS, A.J.A.; SCASE, T.J. et al. Prevalence of hepatic lesions at postmortem examination in dogs and association with pancreatitis. J. Small Anim. Pract., v.51, p.566-572, $2010 \mathrm{~b}$.

XENOULIS, P.G.; STEINER, J.M. Lipid metabolism and hyperlipidemia in dogs. Vet. J., v.183, p.12-21, 2010.

XENOULIS, P.G.; SUCHODOLSKI, J.S.; STEINER, J.M. Chronic pancreatitis in dogs and cats. Comp. Cont. Educ. Vet., v.30, p.166-181, 2008. 